\title{
Reversal of spin polarization in Fe/GaAs (001) driven by resonant surface states: First-principles calculations
}

\author{
Athanasios N. Chantis, ${ }^{1, *}$ Kirill D. Belashchenko, ${ }^{2}$ Darryl L. Smith, ${ }^{1}$ \\ Evgeny Y. Tsymbal, ${ }^{2}$ Mark van Schilfgaarde ${ }^{3}$ and Robert C. Albers ${ }^{1}$ \\ ${ }^{1}$ Theoretical Division, Los Alamos National Laboratory, Los Alamos, New Mexico 87545, USA \\ ${ }^{2}$ Department of Physics and Astronomy and Nebraska Center for Materials and Nanoscience, \\ University of Nebraska-Lincoln, Lincoln, Nebraska 68588, USA \\ ${ }^{3}$ School of Materials, Arizona State University, Tempe, Arizona 85287, USA
}

(Dated: November 30, 2018)

\begin{abstract}
A minority-spin resonant state at the $\mathrm{Fe} / \mathrm{GaAs}(001)$ interface is predicted to reverse the spin polarization with voltage bias of electrons transmitted across this interface. Using a Green's function approach within the local spin density approximation we calculate spin-dependent current in a $\mathrm{Fe} / \mathrm{GaAs} / \mathrm{Cu}$ tunnel junction as a function of applied bias voltage. We find a change in sign of the spin polarization of tunneling electrons with bias voltage due to the interface minority-spin resonance. This result explains recent experimental data on spin injection in Fe/GaAs contacts and on tunneling magnetoresistance in $\mathrm{Fe} / \mathrm{GaAs} / \mathrm{Fe}$ magnetic tunnel junctions.

PACS numbers: 72.25.Mk, 73.23.-b, 73.40.Gk, 73.40.Rw
\end{abstract}

Ferromagnetic metal/nonmagnetic semiconductor contacts have recently attracted significant interest due to the possibility to generate non-equilibrium electron spin distributions in normal semiconductors and hence be practical for spintronics applications [1]. The contact structures in which electron tunneling dominates transport properties are used to achieve a sizable spin polarization of the electric current and produce spin accumulation in the semiconductor. The spin polarization originates from the spin dependence of the wavefunctions and densities of states of the ferromagnetic contact. As a result, the tunneling transmission coefficients are different for majority- and minority-spin electrons.

Among various ferromagnet/semiconductor structures, $\mathrm{Fe} / \mathrm{GaAs}$ contacts have been extensively studied, showing that spin-dependent tunneling through Schottky barriers formed by delta doping is an efficient method for injecting non-equilibrium spin distributions in a semiconductor [2, 3, 4]. These experiments showed that in biased $\mathrm{Fe} / \mathrm{GaAs}$ contacts the net spin of electrons injected from Fe into GaAs is parallel to the magnetization of the ferromagnetic Fe electrode. This implies that majority-spin electrons tunnel through the Schottky barrier more efficiently that minority-spin electrons.

Recently, however, Crooker et al. [5] and Lou et al. [6] observed an anomalous bias dependence of the transport spin polarization in Fe/GaAs Schottky barrier structures. They found that both the magnitude and sign of the spin polarization depend on applied bias voltage producing either majority- or minority-spin accumulation in GaAs. Moser et al. 7] observed a related phenomenon in $\mathrm{Fe} / \mathrm{GaAs} / \mathrm{Fe}$ magnetic tunnel junctions. They found that tunneling magnetoresistance (TMR) changes sign with bias voltage, reflecting the reversal of the transport spin polarization at the $\mathrm{Fe} / \mathrm{GaAs}$ interface. To explain the experiments by Crooker et al. [5] Dery and Sham [8] developed a model suggesting that the reversal of spin polarization is due to localized states at the interface formed by electrostatic confinement of doping profiles. This explanation, however, does not take into account a realistic electronic structure of the interface which is known to be decisive for spin-dependent transport in the tunneling regime [9]. Moreover, it doesn't explain the experiment by Moser et al. [7].

In this Letter, we demonstrate that the observed reversal of the spin polarization in $\mathrm{Fe} / \mathrm{GaAs}(001)$ tunnel contacts is intrinsic to their interface electronic structure. The $\mathrm{Fe} / \mathrm{GaAs}(001)$ interface supports a minorityspin interface band lying in the vicinity of the Fermi energy [10, 11]. This interface band is reminiscent of the $\mathrm{Fe}(001)$ surface band observed experimentally using scanning tunneling spectroscopy [12]. Due to the coupling to continuum bulk states in $\mathrm{Fe}$ the $\mathrm{Fe} / \mathrm{GaAs}(001)$ interface band evolves into an interface resonant band and strongly contributes to the tunneling conductance. The minorityspin character of this resonant band leads to the reversal of the spin polarization from positive to negative in the relevant range of electron energies. This explains the experimental findings of anomalous bias dependence of the spin polarization in experiments on spin injection [5, 6] and TMR [7].

The results of experiments [5, 6, 7] reflect features of spin transmission across the $\mathrm{Fe} / \mathrm{GaAs}(001)$ interface. This is due to the transport spin polarization in tunneling geometry being largely controlled by the interface atomic and electronic structure [9]. In the case of spin injection [5, 6], electrons injected from Fe into GaAs tunnel through the GaAs barrier, then experience scattering by a defect or impurity, and further propagate diffusively producing spin accumulation in GaAs. Since diffusive transport in a nonmagnetic material is independent of electron spin, the spin polarization established within 
GaAs is entirely due to asymmetry in the spin transmission across the $\mathrm{Fe} / \mathrm{GaAs}(001)$ interface. A similar argument applies to spin extraction from GaAs into Fe. In case of magnetic tunnel junctions, variations in TMR are expected to reveal spin polarizations of the two interfaces [13]. However, Moser et al. 7] observed a reversed TMR only for those $\mathrm{Fe} / \mathrm{GaAs} / \mathrm{Fe}$ tunnel junctions in which one interface was "ideal" epitaxial, whereas the other was either oxidized or cleaned by a $H^{+}$plasma. Therefore, their findings reveal features of spin transmission across the epitaxial $\mathrm{Fe} / \mathrm{GaAs}(001)$ interface only.

To study spin-polarized transport across the $\mathrm{Fe} / \mathrm{GaAs}(001)$ interface we consider a Fe/GaAs/Cu(001) tunnel junction with a bcc $\mathrm{Cu}$ counter-electrode, which serves as a detector of spin polarization, in the spirit of Ref. [14]. The bcc $\mathrm{Cu}$ electrode has a spin-independent free-electron-like band structure and a featureless surface transmission function [13], making it a perfect spin detector. This implies that variations in the spin polarization of the tunneling current with bias voltage found in the calculation performed for the $\mathrm{Fe} / \mathrm{GaAs} / \mathrm{Cu}(001)$ tunnel junction are entirely due to the changes in the spin transmission across the $\mathrm{Fe} / \mathrm{GaAs}(001)$ interface. This makes the results of our calculations relevant to experiments [5, 6, 7].

The particular junction studied consists of a semiinfinite Fe electrode, 8 monolayers of GaAs barrier, and a semi-infinite bcc $\mathrm{Cu}$ electrode. We consider an Asterminated interface, motivated by the experiments on spin injection [5, 6] where the epitaxial Fe/GaAs interfaces were grown in As-rich environment [15]. Since intermixing of $\mathrm{Fe}$ and As atoms at this interface is not energetically favorable [11, 16], we assume that the interface is abrupt. The small change of the As-Ga interplane distance of about $0.14 \AA$ due to relaxation [11] is not taken into account.

Calculations are performed using the Green's function representation of the tight-binding linear muffin-tin orbital (TB-LMTO) method in the atomic sphere approximation (ASA) [17]. We apply third-order parametrization for the Green's function [18]. The electronic structure problem is solved within the scalar-relativistic density functional theory (DFT) where the exchange and correlation potential is treated in the local spin-density approximation (LSDA). The conductance is calculated using the principal-layer Green's function technique [19, 20] within the Landauer-Büttiker approach 21]. Charge selfconsistency is achieved before performing the transport calculations.

The spin-dependent transmission coefficient $t^{\sigma}\left(E, \mathbf{k}_{\|}\right)$ is calculated for a given spin $\sigma=\uparrow, \downarrow$ (where $\uparrow$ and $\downarrow$ denote majority and minority spin, respectively) as a function of energy $E$ and the transverse wave vector $\mathbf{k}_{\|}$which is conserved due to the transverse periodicity of the junction. The total transmission for a given energy and spin is obtained by integrating over $\mathbf{k}_{\|}$within a two-dimensional
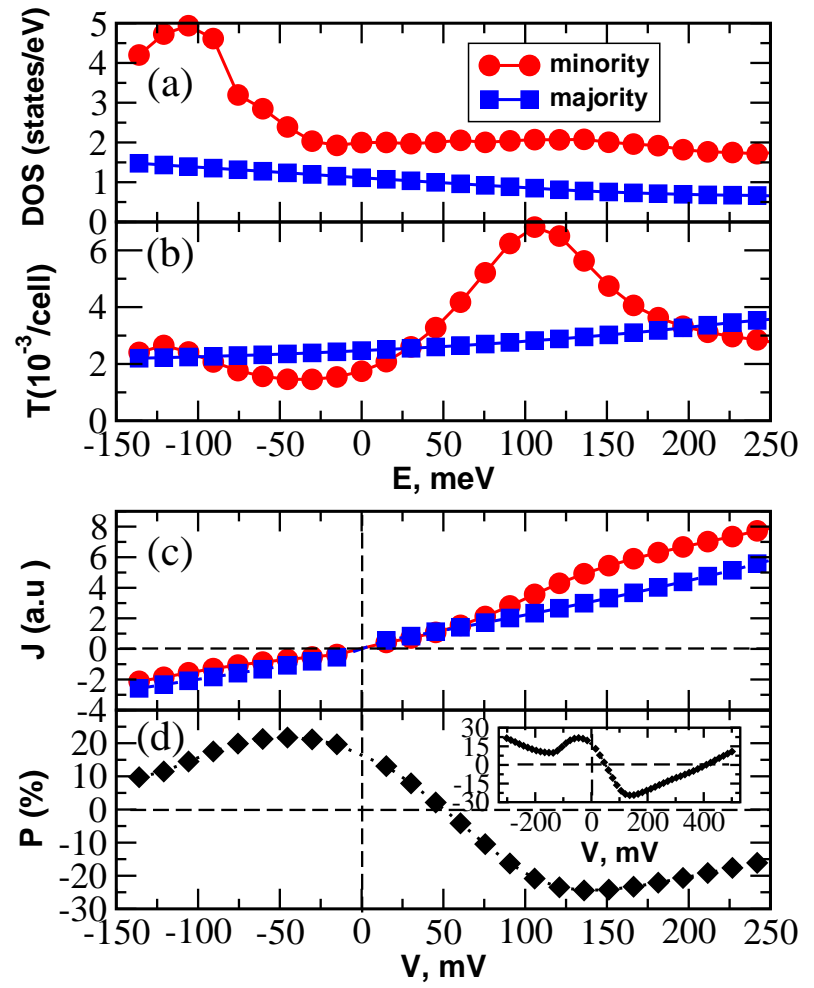

FIG. 1: Results of calculations for a Fe/GaAs/Cu tunnel junction: (a) spin-resolved local DOS for the interface Fe monolayer; (b) spin-resolved integrated transmission as a function of energy; (c) spin-resolved current density as a function of bias voltage; (d) spin polarization as a function of bias voltage. The inset shows the spin polarization over an extended range of bias 22]. In (a) and (b), the Fermi level is set at zero energy.

Brillouin zone (2DBZ): $T^{\sigma}(E)=\int t^{\sigma}\left(E, \mathbf{k}_{\|}\right) d^{2} \mathbf{k}_{\|} /(2 \pi)^{2}$. A uniform $200 \times 200$ mesh is used for the integration. The current density associated with this transmission is obtained from $J^{\sigma}(V)=(e / h) \int_{E_{F}}^{E_{F}+e V} T^{\sigma}(E) d E$, where $E_{F}$ is the Fermi energy and $V$ is the applied bias voltage. This is a reasonable approximation for small voltages considered in this work. This definition of $J^{\sigma}(V)$ implies that for a negative voltage electrons tunnel from $\mathrm{Fe}$ across GaAs. The spin polarization is defined as $P=\left(J^{\uparrow}-J^{\downarrow}\right) /\left(J^{\uparrow}+J^{\downarrow}\right)$.

Figs. 17a and 1b show the calculated local density of states (DOS) at the interface Fe monolayer and the integrated transmission as a function of energy for the $\mathrm{Fe} / \mathrm{GaAs} / \mathrm{Cu}$ junction. The energies are given with respect to $E_{F}$ which is found to be in the middle of the GaAs band gap in agreement with previous calculations 10, 11]. As is seen from Fig. 17, the minority spin dominates the interface DOS in the vicinity of the Fermi energy throughout the energy interval shown. There is a sharp peak in the minority-spin DOS between -50 and $-160 \mathrm{meV}$. The majority-spin transmission (Fig. 1b) exhibits a featureless free-electron-like energy dependence 


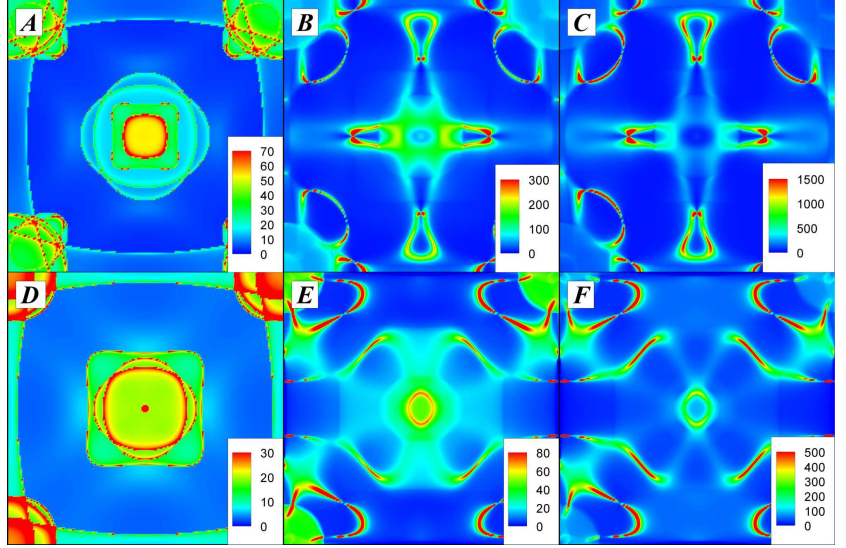

FIG. 2: Minority-spin Fe local density of states resolved in the two-dimensional Brillouin zone by $\mathbf{k}_{\|}$with abscissa along $[\overline{1} \overline{0}]$ and ordinate along $[\overline{0} \overline{1}]$ direction. The upper three panels are for $E=-121 \mathrm{meV}$ corresponding to the local maximum of the minority-spin transmission: (a) bulk, (b) sub-interface monolayer, (c) interface monolayer. The lower three panels are for $E=106 \mathrm{meV}$ corresponding to the maximum of the minority-spin transmission: (d) bulk, (e) sub-interface monolayer, (f) interface monolayer.

mirroring the featureless majority-spin DOS (Fig. 1a). In contrast, the minority-spin transmission is nonmonotonic and dominates in two energy windows, between -130 and $-110 \mathrm{meV}$ and between +50 and $+175 \mathrm{meV}$ (Fig. 1b). The former local maximum corresponds to the sharp peak in the minority-spin interface DOS, whereas the latter maximum has no distinct analog in the DOS.

The energy dependence of the transmission is reflected in the voltage dependence of the spin-resolved current density shown in Fig. 1k. It is seen that, while for negative bias voltages majority-spin electrons dominate the current density, there is a crossover at about +50 $\mathrm{mV}$ which makes the minority-spin current dominating at higher voltages up to $V=+400 \mathrm{mV}$ (see inset in Fig. 1d). This leads to the reversal of spin polarization at about $V=+50 \mathrm{mV}$ seen in Fig. 11. At $V=+400 \mathrm{mV}$ the spin polarization changes sign again reversing from anomalous (negative) to normal (positive). The transmission peak between -130 and $-110 \mathrm{meV}$ (Fig. 1b) is too small to change the sign of the spin polarization and only leads to a reduction of the spin polarization by about $10 \%$. The reversal of the spin polarization with bias voltage is the central result of this Letter. In the following we will show that an interface resonant band is responsible for this anomalous behavior.

Fig. 2 shows the $\mathbf{k}_{\|}$-resolved minority-spin local DOS for two monolayers of $\mathrm{Fe}$ at the Fe/GaAs (001) interface in comparison to the bulk DOS of Fe. The upper three panels correspond to the energy $E=-121 \mathrm{meV}$ at the maximum in the interface DOS (Fig. 1. a) and the matching local peak in the transmission (Fig. 10). The lower three panels correspond to the energy $E=+106 \mathrm{meV}$ at

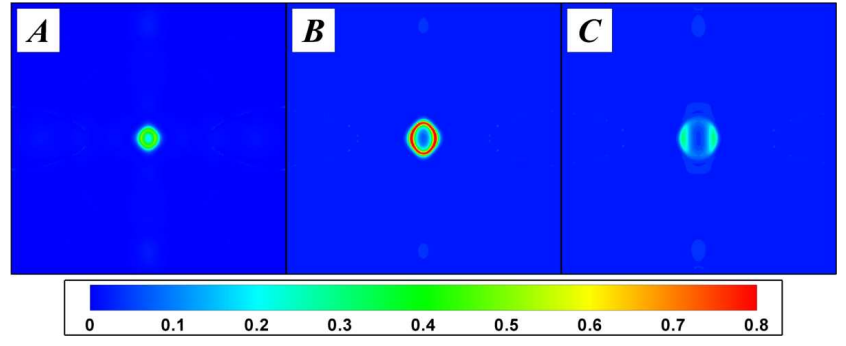

FIG. 3: $\mathbf{K}_{\|}$-resolved minority-spin transmission through a $\mathrm{Fe} / \mathrm{GaAs} / \mathrm{Cu}$ (001) tunnel junction for three energies near the maximum at $E=106 \mathrm{meV}$ : (a) $45 \mathrm{meV}$, (b) $106 \mathrm{meV}$, (c) $166 \mathrm{meV}$.

the maximum in the transmission (Fig. 1b). It is seen that for both energies the interface DOS is strikingly different from the respective bulk DOS (compare Figs. 22 and $2 \mathrm{a}$, and Figs. 2 and $2 \mathrm{~d}$ ). As is evident from Figs. 2 $\mathrm{r}$ and2, for both energies the interface DOS are characterized by features which have the $C_{2 v}$ symmetry intrinsic to the atomic structure of the $\mathrm{Fe} / \mathrm{GaAs}(001)$ interface. The topology of these features is preserved at the subinterface monolayer, but their intensity drops down by a factor of five (compare Figs. 2r and 2 $\mathrm{b}$, and Figs. 2f and 2 ). This behavior clearly points to the presence of minority-spin interface states at energies $E=-121 \mathrm{meV}$ and $E=+106 \mathrm{meV}$. The integral DOS for the state at $E=-121 \mathrm{meV}$ is much higher then that for the state at $E=+106 \mathrm{meV}$ and consequently the former produces the sharp peak in Fig. 1 1 a, whereas the latter is relatively broad.

The analysis of the character of the interface states (bands) shows that they arise from a mixture of $d_{3 z^{2}-r^{2}}$ and $d_{x y}$ orbitals on the interface Fe sites. These states moderately hybridize with bulk Fe minority-spin bands and develop into interface resonances. The latter fact is evident from their finite width that allows these states to be resolved in $\mathbf{k}_{\|}$space (see Figs. 20 and 2f).

The interface resonances contribute to the tunneling conductance. The magnitude of their contribution, however, strongly depends on their distribution across the 2DBZ, because the decay of evanescent states in GaAs depends on $\mathbf{k}_{\|}$. By analyzing the complex band structure of GaAs, Mavropoulos et al. 23] demonstrated that the decay constant $\kappa$ for the evanescent states has a rather deep parabolic global minimum at the $\bar{\Gamma}$ point $\left(\mathbf{k}_{\|}=0\right)$. This feature strongly suppresses the transmission through the resonant states at $E=-121 \mathrm{meV}$, because they are located far from the $\bar{\Gamma}$ point (Fig. 2 c). In contrast, the resonance at $E=+106 \mathrm{meV}$ corresponds to the opening of a parabolic pocket at the $\bar{\Gamma}$ point which is seen as an ellipse in the surface DOS (Fig. 2f); proximity to the $\bar{\Gamma}$ point allows these electrons to tunnel efficiently across the GaAs barrier.

Fig. [3] shows the $\mathbf{k}_{\|}$-resolved transmission for three en- 
ergies around the transmission maximum at $E=+106$ $\mathrm{meV}$. It is seen that around the $\bar{\Gamma}$ point the resonance band is parabolic and anisotropic, reflecting the $C_{2 v}$ symmetry of the interface. Owing to its location near the zone center and a relatively large DOS, this minority-spin band dominates the transmission near $\mathrm{E}=+106 \mathrm{meV}$. For lower energies (Fig. 3a) the resonant band only partially crosses the Fermi level (because of its finite linewidth) providing fewer states to the tunneling current, while for higher energies (Fig. 3r ) the crossings occur for larger $\mathbf{k}_{\|}$ which reduces the resonant band contribution due to $\mathbf{k}_{\|}$ filtering in GaAs. This leads to the dominant contribution of the minority-spin resonant states in the tunneling conductance in a finite energy window (Fig. 1b) and results in the reversal of the spin polarization at bias voltages from +50 to $+400 \mathrm{mV}$ (Fig. 11d).

Our results explain the experimental data on spin injection by Crooker et al. [5] and Lou et al. [6]. In our calculations the reversal of the spin polarization occurs at positive applied bias voltage. This corresponds to electrons incoming from GaAs into unoccupied states of $\mathrm{Fe}$, that is forward applied bias (spin extraction) in the experiments [5, 6]. We find positive spin polarization for negative applied bias voltage, corresponding to electrons incoming from Fe into GaAs, that is reverse applied bias (spin injection) in experiments [5, 6]. These results are in agreement with the experimental data by Lou et al. [6] (samples A and C). We note, however, that the energy of the interface states is sensitive to details of the sample preparation and may be affected by inter-diffusion and disorder resulting in energy shifts of the order of several tenths of an eV. Such a shift may explain why the reversal of the spin polarization occurs when electrons are injected from Fe into GaAs in sample B of Lou et al. [6].

Our results also agree with the TMR data of Moser et al. [7]. They observed a TMR reversal for $\mathrm{Fe} / \mathrm{GaAs} / \mathrm{Fe}$ tunnel junctions with one epitaxial interface and the other one either oxidized or cleaned with $H^{+}$plasma. Since no anomalies are observed when both interfaces are disordered, the reversed TMR is entirely due to the reversal of the spin polarization at the epitaxial interface, which occurs at bias voltages from -90 to about $+400 \mathrm{mV}$. The minimum in TMR is at $V=+50 \mathrm{mV}$ which corresponds to electrons transmitted across GaAs to the epitaxial interface. This is consistent with our results shown in Fig. 1 $\mathrm{d}$. When a sample was annealed at $150^{\circ} \mathrm{C}$ for $1 \mathrm{~h}$ the first reversal of TMR occured at $+20 \mathrm{mV}$ instead of $-90 \mathrm{mV}$, while the shape of the trace remained essentially unchanged [24]. This supports our view concerning the sensitivity of interface states to sample preparation.

In conclusion, we have demonstrated that the minority-spin resonant states at the $\mathrm{Fe} / \mathrm{GaAs}(001)$ interface are responsible for the reversal of spin polarization of electrons transmitted across this interface. This explains experimental data on spin injection in $\mathrm{Fe} / \mathrm{GaAs}$ contacts and on TMR in $\mathrm{Fe} / \mathrm{GaAs} / \mathrm{Fe}$ magnetic tunnel junctions.

We thank Scott Crooker, Paul Crowell and Dieter Weiss for valuable discussions and for sharing with us their experimental results. The work at Los Alamos National Laboratory was supported by DOE Office of Basic Energy Sciences Work Proposal Number 08SCPE973. KDB acknowledges support from the Nebraska Research Initiative and the NSF EPSCoR First Award. EYT thanks the Nanoelectronics Research Initiative and NSF MRSEC for support. MvS was supported by ONR contract N00014-07-1-0479.

* achantis@lanl.gov, Theoretical Division, Los Alamos National Laboratory, Los Alamos, New Mexico 87545, USA

[1] I. Zutic, J. Fabian, and S. D. Sarma, Rev. Mod. Phys. 76, 323 (2004).

[2] A. T. Hanbicki, B. T. Jonker, G. Itskos, G. Kioseoglou, and A. Petrou, Appl. Phys. Lett. 80, 1240 (2002).

[3] A. T. Hanbicki, O. M. J. van 't Erve, R. Magno, G. Kioseoglou, C. H. Li, B. T. Jonker, G. Itskos, R. Mallory, M. Yasar, and A. Petrou, Appl. Phys. Lett. 82, 4092 (2003).

[4] C. Adelmann, X. Lou, J. Strand, C. J. Palmstrom, and P. A. Crowell, Phys. Rev. B 71, 121301 (2005).

[5] S. A. Crooker, M. Furis, X. Lou, C. Adelman, D. L. Smith, C. J. Palmstrom, and P. A. Crowell, Science 309, 5744 (2005).

[6] X. Lou, C. Adelman, S. A. Crooker, E. S. Garlid, J. Zhang, K. S. M. Reddy, S. D. Flexner, C. J. Palmstrom, and P. A. Crowell, Nature Physics 3, 197 (2007).

[7] J. Moser, M. Zenger, C. Gerl, D. Schuh, R. Meier, P. Chen, G. Bayreuther, W. Wegscheider, and D. Weiss, Appl. Phys. Lett. 89, 162106 (2006).

[8] H. Dery and L. J. Sham, Phys. Rev. Lett. 98, 046602 (2007).

[9] E. Y. Tsymbal, K. D. Belashchenko, J. Velev, S. S. Jaswal, M. van Schilfgaarde, I. I. Oleynik, and D. A. Stewart, Prog. Mater. Science 52, 401 (2007).

[10] W. H. Butler, X.-G. Zhang, X. Wang, J. van Ek, and J. M. MacLaren, J. Appl. Phys 81, 5518 (1997).

[11] D. O. Demchenko and A. Y. Liu, Phys. Rev. B 73, 115332 (2006).

[12] J. A. Stroscio, D. T. Pierce, A. Davies, R. J. Celotta, and M. Weinert, Phys. Rev. Lett. 75, 2960 (1995).

[13] K. D. Belashchenko, E. Y. Tsymbal, M. van Schilfgaarde, D. A. Stewart, I. I. Oleynik, and S. S. Jaswal, Phys. Rev. B 69, 174408 (2004).

[14] A. N. Chantis, K. D. Belashchenko, E. Y. Tsymbal, and M. van Schilfgaarde, Phys. Rev. Lett. 98, 046601 (2007).

[15] S. A. Crooker, private communication.

[16] S. C. Erwin, S.-H. Lee, and M. Scheffler, Phys. Rev. B 65, 205422 (2002).

[17] O. K. Andersen, Phys. Rev. B 12, 3060 (1975).

[18] O. Gunnarson, O. Jepsen, and O. K. Andersen, Phys. Rev. B 27, 7144 (1983).

[19] I. Turek, V. Drchal, J. Kudrnovský, M. Šob, and P. Weinberger, Electronic structure of disordered alloys, surfaces and interfaces (Kluwer, 1997).

[20] J. Kudrnovský, V. Drchal, C. Blaas, P. Weinberger, 
I. Turek, and P. Bruno, Phys. Rev. B 62, 15084 (2000).

[21] S. Datta, Electronic transport in mesoscopic systems (Cambridge University Press, 1995), ch. 3.

[22] We note that the bias interval shown here may exceed the range over which the approximation of small bias works well and hence the inset presents a trend rather than a quantitative description.

[23] P. Mavropoulos, N. Papanikolaou, and P. H. Dederichs, Phys. Rev. Lett. 85, 1088 (2000).

[24] Dieter Weiss, private communication. 